Original Research Paper

\title{
Detection of Hepatitis C Virus Resistant Mutants in the era of Direct Acting Antivirals
}

\author{
Livia Maria Gonçalves Rossi and Paula Rahal \\ Department of Biology, Institute of Bioscience, \\ Language and Exact Science, São Paulo State University, São José do Rio Preto, SP, Brazil
}

\author{
Article history \\ Received: 22-12-2014 \\ Revised: $25-12-2014$ \\ Accepted: 05-01-2014 \\ Corresponding Author: \\ Livia Maria Gonçalves Rossi, \\ Department of Biology, \\ Institute of Bioscience, \\ Language and Exact Science, \\ São Paulo State, University, São \\ José do Rio Preto, SP, Brazil \\ Email: liv.rossi@yahoo.com
}

\begin{abstract}
Hepatitis C Virus (HCV) infects millions of people and represents an important public health problem in different regions of the world. Drug resistance is a major challenge for $\mathrm{HCV}$-infection related control. Importantly, the arrival of a plethora of novel, more powerful drugs has revolutionized the field of HCV treatment. Here, we discuss the relevance of identification of $\mathrm{HCV}$ resistant mutants from a clinical standpoint. The advantage and limitations of molecular testing in clinical setting is presented.
\end{abstract}

Keywords: Hepatitis C Virus, Resistant Mutants, Therapy, Direct Acting-Antiviral

\section{Introduction}

Hepatitis C Virus (HCV) infects millions of people worldwide (Lavanchy, 2009; Mohd Hanafiah et al., 2013) and despite being subject of extensive research for more them 25 year (Choo et al., 1989), effective antiviral therapies have just recently been developed (Afdhal et al., 2014a; 2014b; Jacobson et al., 2013; Kowdley et al., 2013; Lawitz et al., 2013a; 2013c; Sulkowski et al., $2014 \mathrm{~b}$ ). HCV is a single-stranded, positive polarity, enveloped RNA flavivirus. The viral genome is $\sim 9.6 \mathrm{~kb}$ in length and contains a single open reading frame encoding for a large polyprotein which is processed by viral and host proteases into three structural proteins and seven nonstructural proteins (Chevaliez and Pawlotsky, 2006; Stanley et al., 2007). HCV is characterized by a high degree of genetic variability. The virus high replication rate and lack of proofreading activity of the viral polymerase (NS5B) are the main contributors to genetic variability (Cruz-Rivera et al., 2013a; 2013b; Preciado et al., 2014). The molecular plasticity of HCV allows for a rapid rearrangement of the intrahost viral population under different selection pressures (Ralston et al., 2011; Von Hahn, 2007). Consequently, HCV genetic variation plays an important role in antiviral drug escape, especially in this era of Direct Acting Antivirals (DAA) (Marascio et al., 2014).

Originally, anti-HCV therapy was based on Interferon (IFN), initially with IFN alpha (IFN $\alpha$ ) in the early 90s and then in combination with Ribavirin (RBV) in 1998. Introduction of pegylated IFN in 2001 increased the likelihood of a Sustained Virological Response (SVR) in combined therapy regimens. The first generation of approved DAA came as a direct result of advancement in the understanding of the HCV life cycle aided by the subgenomic replicons strategies (Lohmann et al., 1999) and the identification of a cell culture infectious clone (Wakita et al., 2005). DAA directly inhibit specific steps in the HCV viral life cycle, particularly targeting the NS3/4A protease, NS5B polymerase and NS5A phosphoprotein, which are indispensable for viral replication. The first-generation of protease inhibitors telaprevir (Vertex, Janssen, Mitsubishi) and boceprevir (Merck) were licensed for use in combination with peg-IFN and RBV in patients infected with HCV genotype 1 in 2011 (Dore et al., 2011). However, these drugs present low genetic barriers to emergence of resistant mutation (Sarrazin et al., 2007; Susser et al., 2009). A second-wave of NS3-4A protease inhibitors have reached phase II or III clinical trials, including Simeprevir (Janssen), already approved in the United States and Europe (Sheridan, 2014). However, these drugs also exhibit a low genetic barrier to resistance mutations and share extensive cross-resistance with telaprevir and boceprevir (Pawlotsky, 2014). Nucleoside/nucleotide analogues, another class of antiHCV drugs, act as substrates for the HCV-RNAdependent RNA polymerase (RdRp). This family of drugs tends to have higher genetic barriers to resistant mutants because selected mutations decreased viral fitness significantly. Sofosbuvir (Gilead) was the first polymerase inhibitor approved by FDA and several other candidates are in phase II clinical trials. Additionally, HCV NS5A protein inhibitors have been also developed (Sulkowski et al., 2014b). The first NS5A inhibitors, Daclatasvir, developed by Bristol-Myers-Squibb 
(Gao et al., 2010), was followed by a number of alternative compounds, such as Ledipasvir (Gilead), Ombitasvir (Abbvie), among others (Table 1). The secondgeneration of NS5A inhibitors is already under development and the arrival of a plethora of improved drugs is expected in the near future. NS5A inhibitors have a dual mechanism of action: (i) Blocking the replication complex and (ii) inhibiting assembly and release of viral particles (Guedj et al., 2013; Pawlotsky, 2013). Resistance mutations conferring resistance to these type of drugs are commonly found in the domain I of the NS5A protein. Not surprisingly, some of these mutations are shared by both first and second generation molecules, e.g., Q30, L31 and Y93 (Fridell et al., 2010; Pawlotsky, 2014). Nonetheless, Daclatasvir exhibits a high genetic barrier to resistance in vivo, where multiple mutations are required to developed a resistant phenotype (Ross-Thriepland and Harris, 2014). Thus, with the arrival of DAA, a new era in the treatment of chronic hepatitis $\mathrm{C}$ has begun and viral eradication with interferon-free, DAA-based therapies is now feasible. The current HCV-treatment options are the following: (i) Simeprevir/faldaprevir/peg/RBV for genotype 1 infected patients, (ii) sofosbuvir/peg/RBV for genotype 1-6 infected patients, (iii) sofosbuvir/RBV (interferonfree) for genotype 1-4 infected patients, (iv) sofosbuvir/simeprevir for genotype 1 patients and (v) sofosbuvir/daclatasvir for genotype 1-3 patients.
So, the relevant question is, does resistance mutation screening in the era of DAA still make sense from a clinical standpoint? Natural occurring HCV resistant mutants commonly take place in the HCV life cycle. The incidence and frequency of resistant variants is variable and depends on a number of factors (Schneider and Sarrazin, 2014). Several studies have shown that resistant mutants at very low frequencies have no impact on treatment response. For instance, treatment-naïve patients with pre-existing resistant mutants prior to treatment achieved similar SVR rates compared to patients with undetected resistant HCV variants (Bartels et al., 2013; Sulkowski et al., 2013). Moreover, treatment response with the first generation of protease inhibitors is independent of pre-existing $\mathrm{HCV}$ resistant mutants if a good response to the peg/RBV-lead backbone is attained. However, among poor peg/RBV responders, those bearing resistant mutants showed lower SVR rates compared to those with sensitive viral populations (Howe et al., 2014).

In vitro studies suggest that sofosbuvir has a pangenotypic activity (Lam et al., 2012). However, SVR rates differ between genotypes showing consistently lower SVR rates for genotype 3 (Lawitz et al., 2013b), Moreover, differences between genotypes and subtypes have also been observed (Lawitz et al., $2013 b$ ). The reasons for these differences are not well understood.

Table 1. Current list of anti-HCV drugs (approved and clinical trials)

\begin{tabular}{|c|c|c|c|c|c|c|}
\hline Compound class & Generation/type & Agent name & Manufacturer & Status & Barrier & GT \\
\hline \multirow{13}{*}{ NS3/4A protease inhibitors } & \multirow[t]{2}{*}{ First-generation, first-wave } & Telaprevir & Janssen & Approved & Low & 1 \\
\hline & & Boceprevir & Merk & Approved & Low & 1 \\
\hline & \multirow[t]{9}{*}{ First-generation, second-wave } & Simeprevir & Tibotec & Approved & Moderate & $1,2,5,6$ \\
\hline & & Faldaprevir & Boehringer Ingelheim & Phase III & Moderate & 1 \\
\hline & & Asunaprevir & Bristol-Myers Squibb & Pending approval & Moderate & 1,4 \\
\hline & & ABT- $450 / \mathrm{r}$ & Abbvie & Phase III & Moderate & 1 \\
\hline & & Danoprevir & Roche & Phase II & High & $1,2,4$ \\
\hline & & Sovaprevir & Achillion & Phase II & Moderate & 1 \\
\hline & & Vedroprevir & Gilead & Phase II & Moderate & 1 \\
\hline & & IDX320 & Idenix & Phase II & Moderate & $1,2,3,4$ \\
\hline & & Vaniprevir & Merck & Phase III & Moderate & 1 \\
\hline & \multirow[t]{2}{*}{ Second-generation } & MK-5172 & Merck & Phase III & Moderate & $1,2,4,5,6$ \\
\hline & & ACH-2684 & Achillion & Phase II & High & $1,2,4,5,6$ \\
\hline \multirow[t]{11}{*}{ NS5B polymerase inhibitors } & \multirow[t]{2}{*}{ Nucleoside inhibitors } & Sofosbuvir & Gilead sciences & Approved & High & $1-6$ \\
\hline & & Mericitabine & Roche & Phase III & High & 1,4 \\
\hline & \multirow[t]{9}{*}{ Non-nucleoside inhibitors } & Deleobuvir & Boehringer Ingelheim & Phase II & & 1 \\
\hline & & GS-9669 & Gilead Sciences & Phase I & & \\
\hline & & Filibuvir & Pfizer & Phase II & & 1 \\
\hline & & VX-222 & Vertex & Phase II & & 1 \\
\hline & & BMS-791325 & Bristol-Myers Squibb & Phase III & & \\
\hline & & Dasabuvir & Abbott & Pending approval & & 1 \\
\hline & & ABT-072 & Abbott & Phase II & & 1 \\
\hline & & Setrobuvir & Roche & Phase II & & 1 \\
\hline & & Tegobuvir & Gilead sciences & Phase II & & 1 \\
\hline \multirow[t]{12}{*}{ NS5A inhibitors } & \multirow[t]{9}{*}{ First-generation } & Daclatasvir & Bristol-Myers Squibb & Phase III & & \\
\hline & & Ledipasvir & Gilead sciences & Phase III & & \\
\hline & & Ombitasvir & AbbVie & Phase III & & \\
\hline & & PPI-668 & Presidio pharmaceuticals & Phase II & & \\
\hline & & PPI-461 & Presidio pharmaceuticals & Phase II & & \\
\hline & & ACH-2928 & Achillion & Phase II & & \\
\hline & & GSK-2336805 & GlaxoSmithKline & Phase II & & \\
\hline & & BMS-824393 & Bristol-Myers Squibb & Phase II & & \\
\hline & & Samatasvir & Idenix pharmaceuticals & Phase II & & \\
\hline & \multirow[t]{3}{*}{ Second-generation } & MK-8742 & Merck & Phase II & & \\
\hline & & ACH-3102 & Achillion & Phase II & & \\
\hline & & GS-5816 & Gilead sciences & Phase II & & \\
\hline
\end{tabular}


HCV mutants conferring resistance to sofosbuvir have been identified in vitro, mainly associated with S282T mutation (Lam et al., 2012). Additionally, in HCV 1a infected patients, viral variants bearing the I434M mutation were shown to be selected by sofosbuvir, leading to relatively low susceptibility.

Clinical trials showed that few patients who relapse after sofosbuvir monotherapy developed the S282T mutation. Importantly, no mutations associated with resistance to sofosbuvir have been detected in patients who showed a relapse after sofosbuvir combination therapy with either RBV or a second DAA. Moreover, no resistance associated with viral breakthrough has been observed in patients treated with any sofosbuvir regimens and robust and rapid SVR is commonly observed. Interestingly, patients who relapse after treatment with sofosbuvir and simeprevir develop Q80K, D168E and I170T mutations but emergence of sofosbuvir resistant mutants has not been reported.

The high genetic barrier for resistance mutants exhibited by sofosbuvir is likely multi-factorial. Fitness of S282T is severely impaired owed to the malfunction of the HCV RdRp activity, resulting in a decreased replication rate of $\sim 90 \%$ in $\mathrm{HCV}$ la and $1 \mathrm{~b}$, resulting in the absence of viral breakthrough.

Relapse after completion of sofosbuvir-based therapy has exclusively been observed in patients treated with sofosbuvir alone or in combination with RBV. However, relapsing has not been reported in patients treated with combinations including a second DAA or Peg-IFNa. Relapse rates are particularly high in HCV 1 and 3infected patients with previous null response to Peg-IFN $\alpha$ and RBV therapy and those with cirrhosis.

Deep-sequencing analysis allows detection of low frequency viral variants at a remarkable sensitivity (CruzRivera et al., 2013b). While these powerful sequencing methods allow for the detection of HCV resistant mutants (Fonseca-Coronado et al., 2012), clinical usefulness of such expensive methods in clinical settings remains intriguing. While molecular characterization of $\mathrm{HCV}$ variants is a valuable tool for molecular epidemiology studies, outbreak investigation and genetic relatedness studies (Goncalves Rossi and Rahal, 2014), its utility for the monitoring of $\mathrm{HCV}$ resistant mutants in clinical settings remains controversial.

\section{Conclusion}

Treatment of HCV has evolved rapidly resulting in improved SVR rates. Telaprevir and boceprevir have now been replaced by sofosbuvir and simeprevir, with improved efficacy and safety; and therefore, identification of protease inhibitor resistance mutants is no longer needed. While detection of Q80K mutation prior to treatment with simeprevir-based regimens might provide some guidance, decision making process should not rely exclusively on the pre-existence of such mutant.
As a result, testing for $\mathrm{Q} 80 \mathrm{~K}$ mutation is not strongly recommended by the American Association for the Study of Liver Diseases/Infectious Diseases Society of America. Particularly now, that the IFN-free therapy is expected to become the standard of care for HCV in the near future.

In conclusion, more studies are required to evaluate the influence of pre-existing or persistent resistant mutations prior treatment on the efficacy of DAAcontaining therapies. Currently, there is no evidence supporting for a general resistance testing before start of HCV-treatment (Schneider and Sarrazin, 2014). In some instances, resistance testing can assist in the decision making process and patient management. e.g., success of treatment among treatment-naïve patients and in patients after treatment failure with peg/RBV depends on the frequency of pre-existing resistant mutants and with sufficient treatment adherence. For simeprevir, the relative high frequency of pre-existing Q80K variants in genotype 1a in European and North American populations is associated with lower SVR and rapid virologic response rates. Therefore, for $\mathrm{HCV}$ 1a infected patients undergoing treatment with simeprevir is important to consider resistance testing for the Q80K mutant. For sofosbuvir, however, no pre-existing mutants are known; and therefore, resistance testing is not indicated.

In general, patients with poor interferon-responses are more likely to develop resistant variants. Importantly, no cross-resistance exists between NS3 and NS5A or NS5B inhibitors. Therefore, resistance testing prior to sofosbuvir-based, interferon-free therapy in conjunction with an NS5a inhibitor is not required.

For HCV 2 and 3 the sofosbuvir-based, interferon-free therapy and $\mathrm{RBV}$, testing for pre-existing resistant variants is not necessary. In experienced patients treated with sofosbuvir and a protease inhibitor have shown to bear the Q80K variants at baseline. Consequently, prior to PI treatment failure, resistance testing may be useful to detect either Q80K and/or other NS3-variants in patients undergoing sofosbuvir/protease inhibitor re-treatment.

In a recent study the use of sofosbuvir and declastavir with/without RBV in HCV 1-3 infected patients showed baseline mutations associated with DCV resistance. Nevertheless, virologic relapse was detected in only one patient (Sulkowski et al., 2014a). Thus, general resistance testing is not recommended in this case.

Resistant testing for HCV might add "too little and too late" information to include it as part of the decision making process and routine clinical testing required in the management of $\mathrm{HCV}$ cases.

\section{Acknowledgement}

L.M.G.R. was supported by CAPES/DS (n. 33004153079P9) Brazil, through the Programa de Pós-Graduação em Microbiologia, Instituto de 
Biociencias, Letras e Ciencias Exatas-IBILCE, Universidade Estadual Paulista "Júlio de Mesquita Filho", UNESP.

\section{Conflict of Interest}

The authors of this manuscript declare no conflict of interest.

\section{Author's Contributions}

All authors equally contributed in this work.

\section{Ethics}

This article is original and contains unpublished material. The corresponding author confirms that all of the other authors have read and approved the manuscript and no ethical issues involved.

\section{References}

Afdhal, N., K.R. Reddy, D.R. Nelson, E. Lawitz and S.C. Gordon et al., 2014a. Ledipasvir and sofosbuvir for previously treated HCV genotype 1 infection. New Eng. J. Med., 370: 1483-1493. DOI: 10.1056/NEJMoa1316366

Afdhal, N., S. Zeuzem, P. Kwo, M. Chojkier and N. Gitlin et al., 2014b. Ledipasvir and sofosbuvir for untreated HCV genotype 1 infection. New Eng. J. Med., 370: 1889-98. DOI: 10.1056/NEJMoa1402454

Bartels, D.J., J.C. Sullivan, E.Z. Zhang, A.M. Tigges and J.L. Dorrian et al., 2013. Hepatitis C virus variants with decreased sensitivity to Direct-Acting Antivirals (DAAs) were rarely observed in DAAnaive patients prior to treatment. J .Virol., 87: 1544-1553. DOI: 10.1128/JVI.02294-12

Chevaliez, S. and J.M. Pawlotsky, 2006. HCV Genome and Life Cycle. In: Hepatitis C Viruses: Genomes and Molecular Biology, Tan, S.L., (Ed.), Norfolk (UK), ISBN-10: 1-904933-20-3.

Choo, Q.L., G. Kuo, A.J. Weiner, L.R. Overby and D.W. Bradley et al., 1989. Isolation of a cDNA clone derived from a blood-borne non-A, non-B viral hepatitis genome. Science, 244: 359-362. DOI: $10.1126 /$ science. 2523562

Cruz-Rivera, M., J.C. Carpio-Pedroza, A. EscobarGutierrez, D. Lozano and A.V. Castaneda et al., 2013a. Rapid hepatitis $\mathrm{C}$ virus divergence among chronically infected individuals. J. Clin. Microbiol., 51: 629-632. DOI: 10.1128/JCM.03042-12

Cruz-Rivera, M., J.C. Forbi, L.H. Yamasaki, C.A.V. Chacon and A. Martinez-Guarneros et al., 2013b. Molecular epidemiology of viral diseases in the era of next generation sequencing. J. Clin. Virol., 57: 378380. DOI: $10.1016 /$ j.jcv.2013.04.021
Dore, G.J., G.V. Matthews, J. Rockstroh and G. Matthews, 2011. Future of hepatitis C therapy: Development of direct-acting antivirals. Curr. Opin. HIV AIDS 6: 508-513. DOI: $10.1097 / \mathrm{COH} .0 \mathrm{~b} 013 \mathrm{e} 32834 \mathrm{~b} 87 \mathrm{f} 8$

Fonseca-Coronado, S., A. Escobar-Gutierrez, K.R. Tovar, M.Y. Cruz-Rivera and P. Rivera-Osorio et al., 2012. Specific detection of naturally occurring hepatitis $\mathrm{C}$ virus mutants with resistance to telaprevir and boceprevir (protease inhibitors) among treatment-naive infected individuals. J. Clin. Microbiol., 50: 281-287. DOI: 10.1128/JCM.05842-11

Fridell, R.A., D. Qiu, C. Wang, L. Valera and M. Gao, 2010. Resistance analysis of the hepatitis $C$ virus NS5A inhibitor BMS-790052 in an in vitro replicon system. Antimicrobial Agents Chem., 54: 36413650. DOI: 10.1128/AAC.00556-10

Gao, M., R.E. Nettles, M. Belema, L.B. Snyder and V.N. Nguyen et al., 2010. Chemical genetics strategy identifies an HCV NS5A inhibitor with a potent clinical effect. Nature, 465: 96-100. DOI: 10.1038 /nature 08960

Goncalves Rossi, L.M. and P. Rahal, 2014. Challenges in molecular epidemiology of hepatitis $\mathrm{C}$ virus. J. Clin. Virol., 60: 174-176. DOI: 10.1016/j.jcv.2014.03.016

Guedj, J., H. Dahari, L. Rong, N.D. Sansone and R.E. Nettles et al., 2013. Modeling shows that the NS5A inhibitor daclatasvir has two modes of action and yields a shorter estimate of the hepatitis $\mathrm{C}$ virus half-life. Proc. Nat. Acad. Sci. USA, 110: 39913996. DOI: 10.1073/pnas.1203110110

Howe, A.Y., J. Long, D. Nickle, R. Barnard and S. Thompson et al., 2014. Long-term follow-up of patients receiving boceprevir for treatment of chronic hepatitis C. Antiviral Res., 113: 71-78. DOI: 10.1016/j.antiviral.2014.10.010

Jacobson, I.M., S.C. Gordon, K.V. Kowdley, E.M. Yoshida and M. Rodriguez-Torres et al., 2013. Sofosbuvir for hepatitis C genotype 2 or 3 in patients without treatment options. New Eng. J. Med., 368: 1867-1877. DOI: 10.1056/NEJMoa1214854

Kowdley, K.V., E. Lawitz, I. Crespo, T. Hassanein and M.N. Davis et al., 2013. Sofosbuvir with pegylated interferon alfa-2a and ribavirin for treatment-naive patients with hepatitis $\mathrm{C}$ genotype-1 infection (ATOMIC): An open-label, randomised, multicentre phase 2 trial. Lancet, 381: 2100-2107. DOI: 10.1016/S0140-6736(13)60247-0

Lam, A.M., C. Espiritu, S. Bansal, H.M.M. Steuer and C. Niu et al., 2012. Genotype and subtype profiling of PSI-7977 as a nucleotide inhibitor of hepatitis $C$ virus. Antimicrobial Agents Chem., 56: 3359-3368. DOI: 10.1128/AAC.00054-12 
Lavanchy, D., 2009. The global burden of hepatitis C. Liver Int. 29 Suppl., 1: 74-81. DOI: $10.1111 / \mathrm{j} .1478-3231.2008 .01934 . x$

Lawitz, E., A. Mangia, D. Wyles, M.R. Torres and T. Hassanein et al., 2013b. Sofosbuvir for previously untreated chronic hepatitis $\mathrm{C}$ infection. New Eng. J. Med., 368: 1878-1887.

DOI: 10.1056/NEJMoa1214853

Lawitz, E., J.P. Lalezari, T. Hassanein, K.V. Kowdley and F.F. Poordad et al., 2013a. Sofosbuvir in combination with peginterferon alfa- $2 \mathrm{a}$ and ribavirin for noncirrhotic, treatment-naive patients with genotypes 1, 2 and 3 hepatitis $\mathrm{C}$ infection: A randomised, doubleblind, phase 2 trial. Lancet Infect Dis., 13: 401-408. DOI: 10.1016/S1473-3099(13)70033-1

Lawitz, E., M. Sulkowski, I. Jacobson, W.K. Kraft and B. Maliakkal et al., 2013c. Characterization of vaniprevir, a hepatitis $\mathrm{C}$ virus NS3/4A protease inhibitor, in patients with HCV genotype 1 infection: safety, antiviral activity, resistance and pharmacokinetics. Antiviral Res., 99: 214-220. DOI: 10.1016/j.antiviral.2013.05.015

Lohmann, V., F. Korner, J. Koch, U. Herian and L. Theilmann et al., 1999. Replication of subgenomic hepatitis $\mathrm{C}$ virus RNAs in a hepatoma cell line. Science, 285: 110-113.

DOI: $10.1126 /$ science. 285.5424 .110

Marascio, N., C. Torti, M. Liberto and A. Foca, 2014. Update on different aspects of HCV variability: Focus on NS5B polymerase. BMC Infect. Dis. DOI: $10.1186 / 1471-2334-14-S 5-S 1$

Mohd Hanafiah, K., J. Groeger, A.D. Flaxman and S.T. Wiersma, 2013. Global epidemiology of hepatitis $\mathrm{C}$ virus infection: New estimates of agespecific antibody to HCV seroprevalence. Hepatology, 57: 1333-1342. DOI: 10.1002/hep.26141

Pawlotsky, J.M., 2013. NS5A inhibitors in the treatment of hepatitis C. J. Hepatol., 59: 375-382. DOI: $10.1016 /$ j.jhep.2013.03.030

Pawlotsky, J.M., 2014. New hepatitis C therapies: The toolbox, strategies and challenges. Gastroenterology, 146: 1176-1192. DOI: 10.1053/j.gastro.2014.03.003

Preciado, M.V., P. Valva, A. Escobar-Gutierrez, P. Rahal and K. Ruiz-Tovar et al., 2014. Hepatitis C virus molecular evolution: Transmission, disease progression and antiviral therapy. World J. Gastroenterol., 20: 15992-16013. DOI: $10.3748 /$ wjg.v20.i43.15992

Ralston, R., I. Jacobson and M. Scull, 2011. The conundrum of relapse in STAT-C therapy: Does $\mathrm{HCV}$ play the red queen or rip van winkle? Semin. Liver Dis., 31: 410-419.

DOI: $10.1055 / \mathrm{s}-0031-1297929$
Ross-Thriepland, D. and M. Harris, 2014. Hepatitis C virus NS5A: Enigmatic but still promiscuous 10 years on! J. Gen. Virol. DOI: 10.1099/jgv.0.000009

Sarrazin, C., T.L. Kieffer, D. Bartels, B. Hanzelka and U. Muh et al., 2007. Dynamic hepatitis C virus genotypic and phenotypic changes in patients treated with the protease inhibitor telaprevir. Gastroenterology, 132: 1767-1777.

DOI: $10.1053 /$ j.gastro.2007.02.037

Schneider, M.D. and C. Sarrazin, 2014. Antiviral therapy of hepatitis C in 2014: Do we need resistance testing? Antiviral Res., 105: 64-71. DOI: $10.1016 /$ j.antiviral.2014.02.011

Sheridan, C., 2014. FDA approvals usher in the postinterferon era in HCV. Nat. Biotechnol., 32: 3-5. DOI: $10.1038 /$ nbt0114-3

Stanley, M.L., C. Walker, M.J. Alter and M. Yi, 2007. Hepatitis C Virus. In: Fields Virology, Knipe, D.M. and P.M. Howley, (Eds.), Lippincott Williams and Wilkins, Philadelphia. pp: 1253-1304.

Sulkowski, M.S., D.F. Gardiner, M. Rodriguez-Torres, K.R. Reddy and T. Hassanein et al., 2014a. Daclatasvir plus sofosbuvir for previously treated or untreated chronic HCV infection. New Eng. J. Med., 370: 211221. DOI: 10.1056/NEJMoa1306218

Sulkowski, M.S., I.M. Jacobson and D.R. Nelson, 2014b. Daclatasvir plus sofosbuvir for HCV infection. New Eng. J. Med., 370: 1560-1561. DOI: $10.1056 /$ NEJMc1401726

Sulkowski, M.S., T. Asselah, J. Lalezari, P. Ferenci and H. Fainboim et al., 2013. Faldaprevir combined with pegylated interferon alfa- $2 \mathrm{a}$ and ribavirin in treatment-naive patients with chronic genotype 1 HCV: SILEN-C1 trial. Hepatology, 57: 2143-2154. DOI: $10.1002 /$ hep. 26276

Susser, S., C. Welsch, Y. Wang, M. Zettler and F.S. Domingues et al., 2009. Characterization of resistance to the protease inhibitor boceprevir in hepatitis C virus-infected patients. Hepatology, 50: 1709-1718. DOI: 10.1002/hep.23192

Von Hahn, T., J.C. Yoon, H. Alter, C.M. Rice and B. Rehermann et al., 2007. Hepatitis C virus continuously escapes from neutralizing antibody and $\mathrm{T}$-cell responses during chronic infection in vivo. Gastroenterology, 132: 667-678. DOI: $10.1053 /$ j.gastro.2006.12.008

Wakita, T., T. Pietschmann, T. Kato, T. Date and M. Miyamoto et al., 2005. Production of infectious hepatitis $\mathrm{C}$ virus in tissue culture from a cloned viral genome. Nat. Med., 11: 791-796.

DOI: $10.1038 / \mathrm{nm} 1268$ 